\title{
SIMULAÇÃO DA DEGRADAÇÃO MECÂNICA DE PELOTAS DE MINÉRIO DE FERRO EM FORNO DE REDUÇÃO DIRETA*
}

Fernando O. Boechat ${ }^{1}$

Rodrigo M. de Carvalho Anderson W. Baltazar ${ }^{3}$

Resumo

Luís Marcelo Tavares ${ }^{4}$

Com a redução da oferta de minério granulado de boa qualidade no mercado mundial e as restrições ambientais à sinterização, as pelotas de minério de ferro passaram a ocupar um papel importante como matéria prima para produção de ferro primário. Entretanto, embora apresentem maior resistência à degradação, as pelotas ainda sofrem com este problema, embora em menor magnitude, quando comparadas com granulados. Pode-se identificar a degradação das pelotas de minério de ferro em duas etapas, durante o manuseio e sob redução. Uma vez que vários estudos já analisaram a degradação durante o manuseio, a degradação durante a redução torna-se um assunto relevante e pouco explorado. Assim, o presente trabalho avaliou as energias envolvidas no movimento da carga ao longo de um forno de redução direta do tipo Midrex, usando o método dos elementos discretos. Para isso, foi utilizado o software EDEM ${ }^{\circledR}$ para obtenção das energias dissipadas durante as colisões das partículas. Aplicando estas energias ao modelo mecanicista proposto por Tavares e Carvalho [4] para fragmentação das partículas foi possível estimar a taxa de geração de finos no interior do forno. Estes resultados foram utilizados na previsão da degradação mecânica de pelotas no interior do forno, que previu a geração de 1,2 a 2,6\% de finos gerados em um forno Minimod ${ }^{\circledR}$, dependendo das premissas usadas.

Palavras-chave: Pelotas; DEM; Degradação; Redução Direta.

\section{SIMULATION OF MECHANICAL DEGRADATION OF IRON ORE PELLETS INSIDE A DIRECT REDUCTION FURNACE}

\section{Abstract}

The reduced supply of good quality lump ore and environmental constraints to sintering changed the participation of iron ore pellets in the world market becoming an important raw material for production of primary iron. However, while presenting greater resistance to degradation, pellets still suffer from this problem, although to a lesser magnitude compared to lump ore. It is possible to classify degradation of iron ore pellets in two stages: during handling and under reduction. Since several studies explain the degradation during handling, its degradation during reduction becomes an interesting object of study. As such, the present work estimated the energies involved in the movement of pellets along a Midrex direct reduction furnace using the discrete element method. The software EDEM ${ }^{\circledR}$ was used to obtaining the dissipated energies during collisions of the particles. Applying these energies in the mechanistic model proposed by Tavares and Carvalho [4] for particle fragmentation it was possible to determine the rate of formation of fine inside the reactor. These results were used to estimate the mechanical degradation of pellets in the furnace, which resulted in estimates that varied from 1.2 to $2.6 \%$ of fines in a Minimod ${ }^{\circledR}$ furnace, depending on the assumptions used.

Keywords: Pellets; DEM; Degradation; Direct Reduction.

1 M.Sc em Eng. Metalúrgica e de Materiais, Programa de Engenharia Metalúrgica e de Materiais, COPPE, Universidade Federal do Rio de Janeiro, Rio de Janeiro, RJ, Brasil.

2 D.Sc. em Eng. Metalúrgica e de Materiais, Programa de Engenharia Metalúrgica e de Materiais, COPPE, Universidade Federal do Rio de Janeiro, Rio de Janeiro, RJ, Brasil.

3 D.Sc. em Eng. Metalúrgica e de Materiais, Centro de Tecnologia de Ferrosos, Vale S.A., Nova Lima, MG, Brasil.

4 Ph.D. em Eng. Metalúrgica, Programa de Engenharia Metalúrgica e de Materiais, COPPE, Universidade Federal do Rio de Janeiro, Rio de Janeiro, RJ, Brasil.

* Contribuição técnica ao $44^{\circ}$ Seminário de Redução de Minério de Ferro e Matérias-primas, $15^{\circ}$ Simpósio Brasileiro de Minério de Ferro e $2^{\circ}$ Simpósio Brasileiro de Aglomeração de Minério de Ferro, 15 a 18 de setembro de 2014, Belo Horizonte, MG, Brasil. 


\section{INTRODUÇÃO}

Com a redução da oferta de minério granulado de boa qualidade no mercado mundial e a restrições ambientais a sinterização, as pelotas de minério de ferro passaram a ocupar um papel importante como matéria prima para produção de ferro primário. Sua participação na produção de ferro gusa e ferro esponja têm aumentado ano após ano, devido às suas características vantajosas quando comparadas ao minério granulado. A sua homogeneidade granulométrica, a sua constância de qualidade química, o seu baixo nível de degradação física durante o manuseio e sob-redução são algumas das características que tem atraído produtores de ferro primário para a sua aplicação.

O aumento na produção de aço via forno elétrico a arco impactou diretamente na produção de ferro esponja que saltou de $40 \mathrm{Mt}$ no início do século para $74 \mathrm{Mt}$ em 2012 [1]. Diversos processos são utilizados na produção de ferro esponja, no entanto o maior destaque está no processo MIDREX (Midland Ross Experimental) que correspondeu a $60,5 \%$ da produção mundial de ferro esponja em 2012 [3]. Neste processo é usado o minério granulado e/ou pelotas de minério de ferro como carga metálica. As vantagens apresentadas pelas pelotas de minério de ferro contribuem para melhorar a estabilidade operacional e para atingir ganhos de produtividade e/ou metalização, tornando esta carga mais atrativa para os produtores de ferro esponja via fornos MIDREX [2].

Entretanto, embora apresentem maior resistência à degradação, as pelotas ainda sofrem com este problema, embora em menor magnitude. Pode-se identificar a degradação das pelotas de minério de ferro em duas etapas: durante seu manuseio e sob redução. Durante o manuseio, logo após sua fabricação até o processo de redução, as pelotas são sujeitas a diferentes esforços que, dependendo de diversos fatores durante sua fabricação, poderá leva-las a se fragmentar. A fim de prever a proporção de finos, bem como a proporção de fragmentos (cacos), gerados durante esta etapa, foi desenvolvido um modelo matemático por Tavares e Carvalho [4] que permite a previsão da degradação de granéis quando submetidos a impactos, como aqueles que ocorrem durante 0 manuseio. Estes finos são retirados por peneiramento antes da carga ser inserida no forno de redução direta. A partir deste momento, entretanto, ocorrerá a degradação sob redução. A fragmentação será resultado da fragilização da estrutura da pelota pela redução da resistência mecânica durante a sua redução tanto devido a alterações na sua microestrutura quanto devido às condições às quais o óxido de ferro estará sujeito podendo gerar finos e fragmentos. Esta ocorrência implica em perda de produtividade do forno pelo fato que a geração destes finos e cacos prejudicam a permeabilidade do leito de pelotas. Isso, por sua vez, resulta na redução da metalização da carga ou no aumento do tempo de residência da carga no forno, com redução da produtividade do forno. Desta forma, a determinação da quantidade de finos que será gerado no interior do reator devido ao uso de determinado tipo de pelota pode auxiliar na otimização da carga ou da mistura de componentes (mix) a ser usado no reator de redução direta.

\section{MATERIAIS E MÉTODOS}

\subsection{Experimental}

A simulação em DEM (Discrete Element Method) da operação em um forno de redução pode fornecer as informações necessárias para simulação da degradação

\footnotetext{
* Contribuição técnica ao $44^{\circ}$ Seminário de Redução de Minério de Ferro e Matérias-primas, 15은 Simpósio Brasileiro de Minério de Ferro e 2o Simpósio Brasileiro de Aglomeração de Minério de Ferro, 15 a 18 de setembro de 2014, Belo Horizonte, MG, Brasil.
} 
do material particulado também nesse tipo de equipamento. Isto torna possível prever se há severidade suficiente nesta operação para geração de finos no interior do forno. Pela simulação em DEM é possível analisar o movimento durante a redução da carga pelo qual se podem gerar forças de contatos entre as pelotas capazes de degradar a carga, sem levar em consideração as forças resultantes da redução do minério. Quanto maior a proporção de finos e cacos de pelotas formados, menor será a permeabilidade no interior do forno e mais favorecida será a formação de cachos. Desta forma, a simulação da operação (movimento no interior do forno) em DEM pode fornecer informações das energias envolvidas nos contatos das pelotas para posterior aplicação do modelo de quebra previamente desenvolvido por Tavares e Carvalho [4].

Para que seja simulada a degradação no interior do forno, é necessário prever a distribuição completa de tamanhos de partículas após cada impacto, não apenas a proporção de material original que é fragmentado. A equação geral do balanço de massas (Equação 01) é obtida a partir do balanço de cada fração de tamanho após uma queda individual de todas as partículas:

$$
w_{i, n+1}=w_{i, n}\left[1-F_{i, n}\left(e E_{k_{i}, n}\right)\right]\left(1-k_{i}\right)+\sum_{j=1}^{i} w_{j, n}\left[F_{j, n}\left(e E_{k, n}\right) b_{i j}\left(e E_{k, n}\right)+k_{j}\left[1-F_{j, n}\left(e E_{k_{s}, n}\right)\right] a_{i j}\right]
$$

na qual $w_{i}$ é a fração de partículas na classe de tamanho $i, n$ é o número do evento de colisão, $F_{i}$ é a distribuição de energias de fratura das partículas de tamanho $i$, eE $E_{k}$ é a energia efetiva recebida pelas partículas, $b_{i j}$ é função quebra das partículas (distribuição dos fragmentos), $k_{j}$ é a proporção de material degradado por abrasão e $a_{\mathrm{ij}}$ é a função quebra das partículas degradadas por abrasão. Essa função é descrita pela relação [5]:

$$
k=a\left(\frac{E_{k}^{k}}{E_{50 u}}\right)^{\gamma}
$$

sendo que E50u é a energia de fratura das partículas que não quebraram, $\gamma$ e a são parâmetros de ajuste a serem calibrados a partir de dados experimentais.

Esta suscetibilidade à fratura superficial (abrasão) pode ser descrita a partir de resultados de ensaios de queda livre de pelotas a diferentes alturas e ângulos de impacto, registrando-se a proporção de finos $k$ gerada (Figura ). Ajustando-se os parâmetros da Equação 02, tem-se:

$$
k=0,002 E_{k}^{1,1623}
$$

na qual a energia efetiva de impacto $E_{k}$, em $\mathrm{J} / \mathrm{kg}$, é obtida pela composição da energia normal $E_{n}$ e tangencial $E_{t}$, tal que [6]

$$
E_{k}=E_{n}+0,2 E_{t}
$$




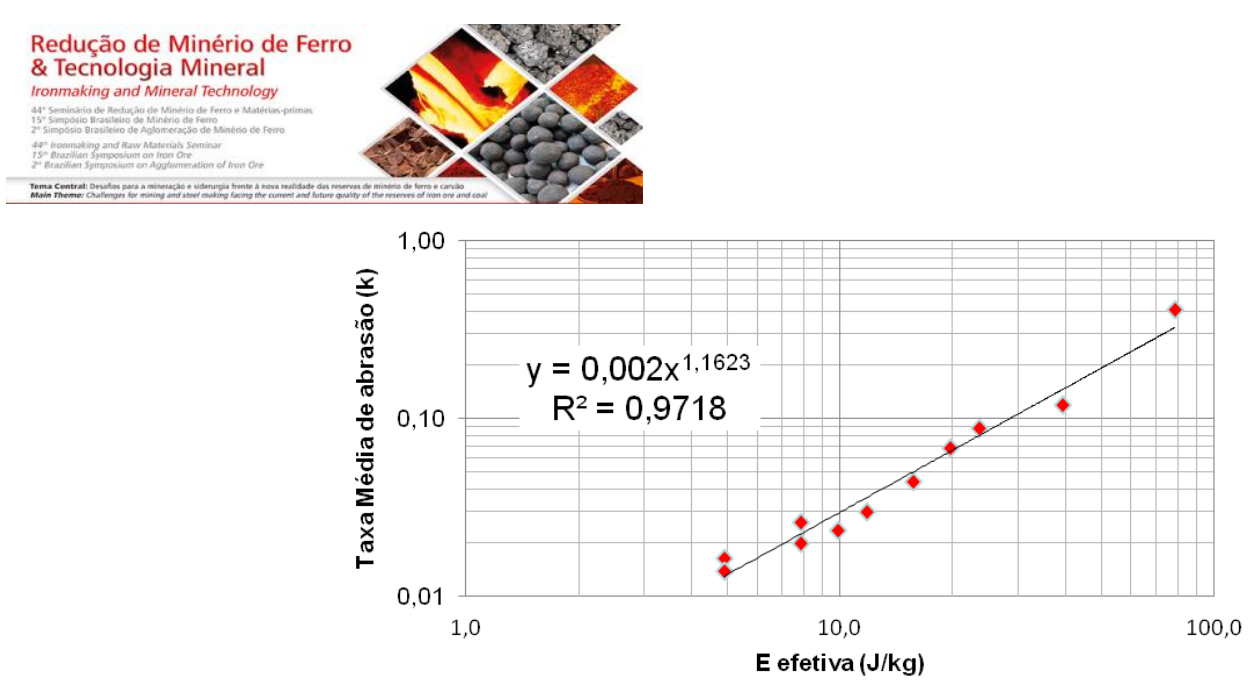

Figura 1 - Resultados experimentais para calibração da proporção de finos gerados.

Após cada impacto, a distribuição de energias de fratura, específica em relação à massa, das partículas contidas em cada classe de tamanho $i$ deve ser modificada, uma vez que algumas partículas contidas nesta classe de dimensão podem ter sido quebradas e as partículas que permaneceram integras podem ter enfraquecido. Complementando, os fragmentos resultantes da quebra de partículas mais grossas podem ter passado para este tamanho i. A distribuição de energias de fratura das partículas contidas na classe de tamanhos $i$ após o evento de impacto $n$ é dada por:

$$
F_{i, n+1}(E)=\frac{F_{i, n+1}^{*}(E) w_{i, n}\left\lfloor 1-F_{i, n}\left(E_{k, n}\right)\right\rfloor\left(1-k_{i}\right)+F_{i, 0}(E) \sum_{j=1}^{i} w_{i, n}\left\lfloor F_{j, n}\left(E_{k, n}\right) b_{i, j}+\left\lfloor 1-F_{j, n}\left(E_{k, n}\right)\right\rfloor k_{j} a_{i}\right\rfloor}{w_{i, n+1}}
$$

na qual $F_{i, n+1}^{*}(E)$ é a distribuição de energias das partículas que sofrem dano (recebem impacto), mas não fraturam, e $F_{i, 0}$ é a distribuição de energias das partículas originais, obtida a partir da compressão individual de pelotas em laboratório.

\subsection{Modelagem e Simulação}

Os critérios utilizados nas simulações foram estabelecidos com o objetivo de minimizar o tempo de processamento em cada etapa da simulação. Como uma das limitações atuais ao uso do DEM está no processamento computacional, cuidados foram tomados na construção do modelo de forma a reduzir ao máximo o número de partículas no sistema, a utilizar-se um formato e tamanho de partícula adequado, a escolher as corretas propriedades dos materiais e determinar o tempo mínimo a ser simulado.

Para a construção do modelo do forno de redução foi utilizado o software baseado em DEM, o EDEM ${ }^{\circledR}$. O forno de redução simulado apresentou dimensões e geometria equivalentes a um forno MIDREX do tipo Minimod ${ }^{\circledR}$. A escolha deste forno teve como premissa a utilização de um forno de tamanho industrial e ao mesmo tempo, reduzir ao máximo as dimensões a serem simuladas, sem haver perda do fator escala. Com vistas a reduzir o número de partículas dentro da simulação, 0 forno foi dividido em quatro regiões, as quais foram simuladas individualmente. As regiões e números de partículas envolvidas em cada simulação podem ser observados na Figura 2. 


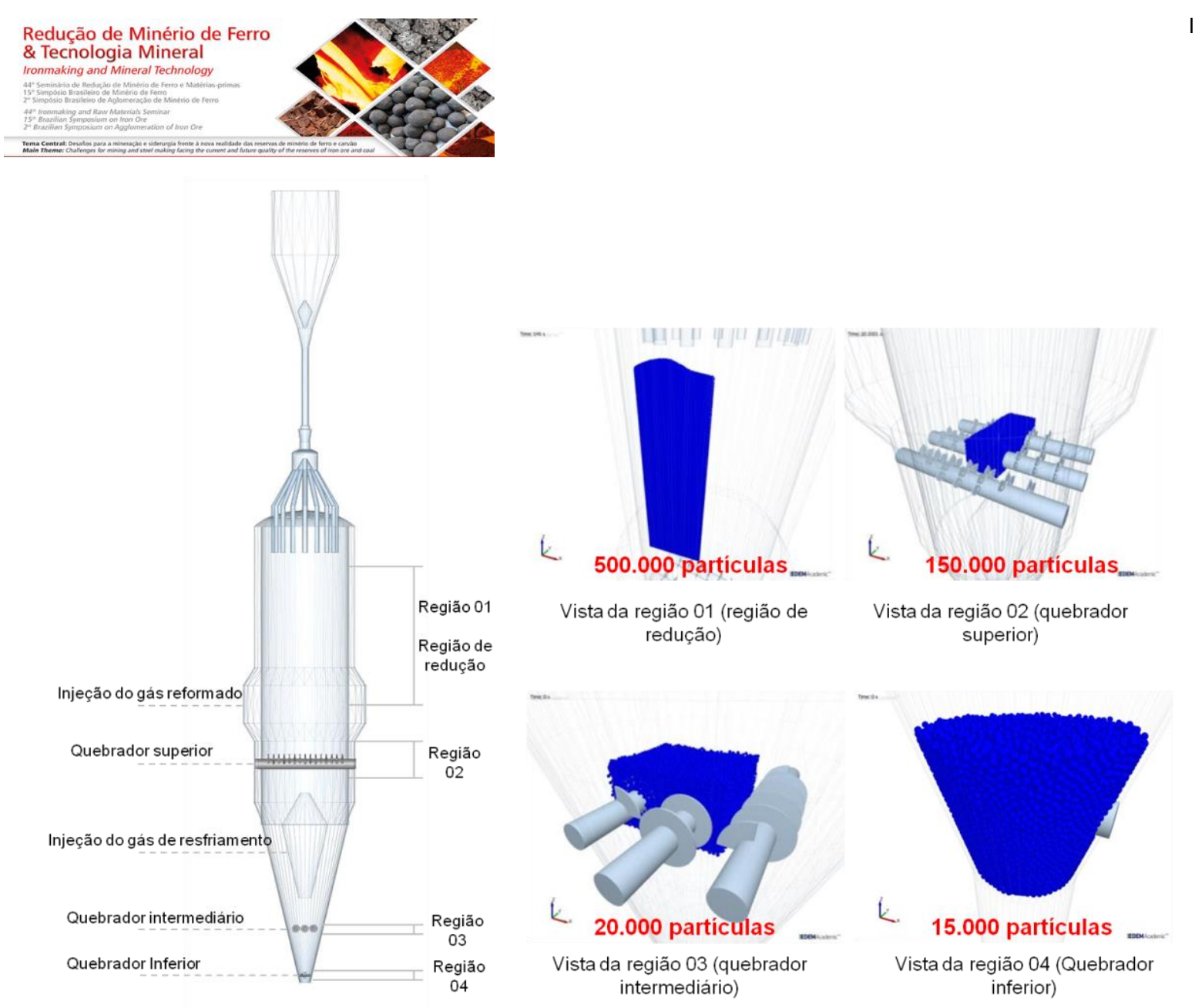

Figura 1 - Regiões simuladas do reator MIDREX.

Para descrever o contato das partículas foi considerando o modelo de contato de Hertz-Mindlin, disponível no EDEM ${ }^{\circledR}$. As pelotas foram utilizadas no formato esférico e com tamanho de $25 \mathrm{~mm}$, resultando no número de partículas apresentadas na Figura 2. Este tamanho representa, aproximadamente, o dobro do tamanho médio de pelotas de minério de ferro utilizadas industrialmente, mas a sua escolha foi necessária a fim de diminuir o número de partículas nas simulações. A massa específica das pelotas e demais parâmetros de contato foram determinados previamente em Barrios et al. (

Tabela).

Tabela 1 - Parâmetros de contato descritos por Barrios et al. para pelotas com formato esférico

\begin{tabular}{lcccc}
\hline \hline & Aço & Pelota & Pelota/Pelota & Pelota/Aço \\
\hline \hline Coeficiente de Poisson & 0,3 & 0,25 & & \\
\hline Modulo de cisalhamento $(\mathrm{MPa})$ & 7000 & 16 & & \\
\hline Densidade $\left(\mathrm{kg} / \mathrm{m}^{3}\right)$ & 7800 & 3948 & & \\
\hline
\end{tabular}

\begin{tabular}{lll}
\hline Coeficiente de restituição & 0,49 & 0,5 \\
\hline Coeficiente de Atrito estático & 0,21 & 0,25 \\
\hline $\begin{array}{l}\text { Coeficiente de Atrito por } \\
\text { rolamento }\end{array}$ & 0,48 & 0,39 \\
\hline \hline
\end{tabular}

A velocidade de descida da carga para cada região foi determinada com base na produção do reator simulado de $16,1 \mathrm{~kg} / \mathrm{s}$, o que corresponde a uma capacidade produtiva anual da ordem de 500.00 toneladas. Os três sistemas de quebradores

* Contribuição técnica ao 44 Seminário de Redução de Minério de Ferro e Matérias-primas, $15^{\circ}$ Simpósio Brasileiro de Minério de Ferro e $2^{\circ}$ Simpósio Brasileiro de Aglomeração de Minério de Ferro, 15 a 18 de setembro de 2014, Belo Horizonte, MG, Brasil. 
foram inseridos nos modelos e as suas dimensões e modo de funcionamento destas partes móveis do forno foram utilizadas com base nos parâmetros operacionais, descritos na Tabela.

As simulações foram realizadas de forma a acompanhar o fluxo de pelotas pelo sistema de alimentação e os dados para análise foram extraídos após o fluxo entrar em regime estacionário. As simulações foram pós-processadas utilizando o LTM Analyst 2013 [6].

Tabela 2- Parâmetros operacionais utilizados para os quebradores

\begin{tabular}{lccccc}
\hline Posição & $\begin{array}{c}\text { Ângulo de } \\
\text { rotação }\left({ }^{\circ}\right)\end{array}$ & Tempo $(\mathrm{s})$ & $\begin{array}{c}\text { Frequência } \\
(\mathrm{rad} / \mathrm{s})\end{array}$ & $\begin{array}{c}\text { Raio do eixo } \\
(\mathrm{m})\end{array}$ & $\begin{array}{c}\text { Raio do } \\
\text { quebrador } \\
(\mathrm{m})\end{array}$ \\
\hline Superior & 45 & 180 & 0,00436 & 0,228 & 0,487 \\
Intermediário & 90 & 30 & 0,05236 & 0,080 & 0,175 \\
Inferior & 60 & 10 & 0,10472 & 0,069 & 0,160 \\
\hline
\end{tabular}

A simulação da geração de finos foi feita pela aplicação da Equação 03 com base nos dados de energias dissipadas durante as colisões obtidas nas simulações realizadas no EDEM. Entretanto a Equação 03 descreve a geração de finos considerando os parâmetros de pelotas antes da redução. Para poder avaliar de perda de resistência superficial que ocorre nas pelotas durante sua redução e com isto, sua maior suscetibilidade a abrasão, foram utilizadas as informações contidas nos trabalhos de Huang et al. [7] e Parisi e Laborde [8]. Nestes trabalhos foi analisada a perda da resistência das pelotas de minério de ferro, devido sua redução, e modelada a redução relativa à altura da carga, respectivamente. Com base nos dados destes dois trabalhos foi possível estimar uma relação entre a resistência superficial da pelota com sua altura na região de redução do forno (Figura 3).

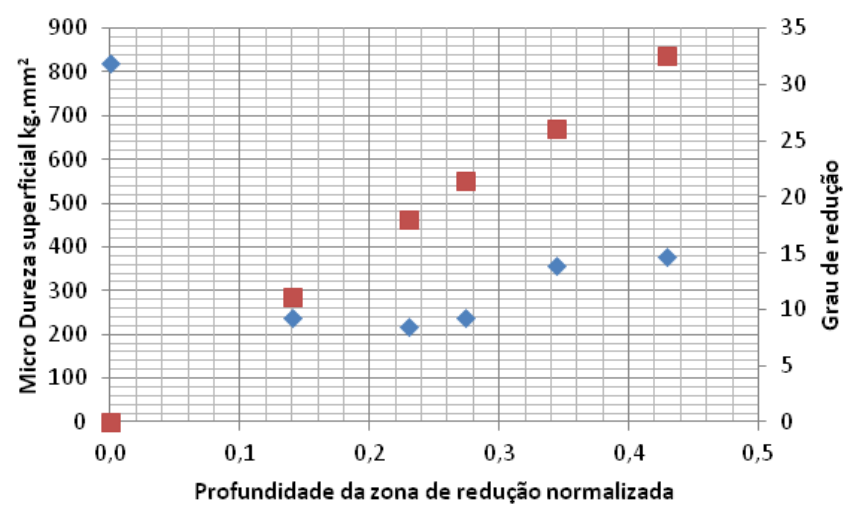

Figura 3 - Variação da micro dureza superficial de pelotas e do grau de redução em função da posição de pelotas no interior de forno (dados de HUANG et al., 2012 e PARISI e LABORDE, 2004) 

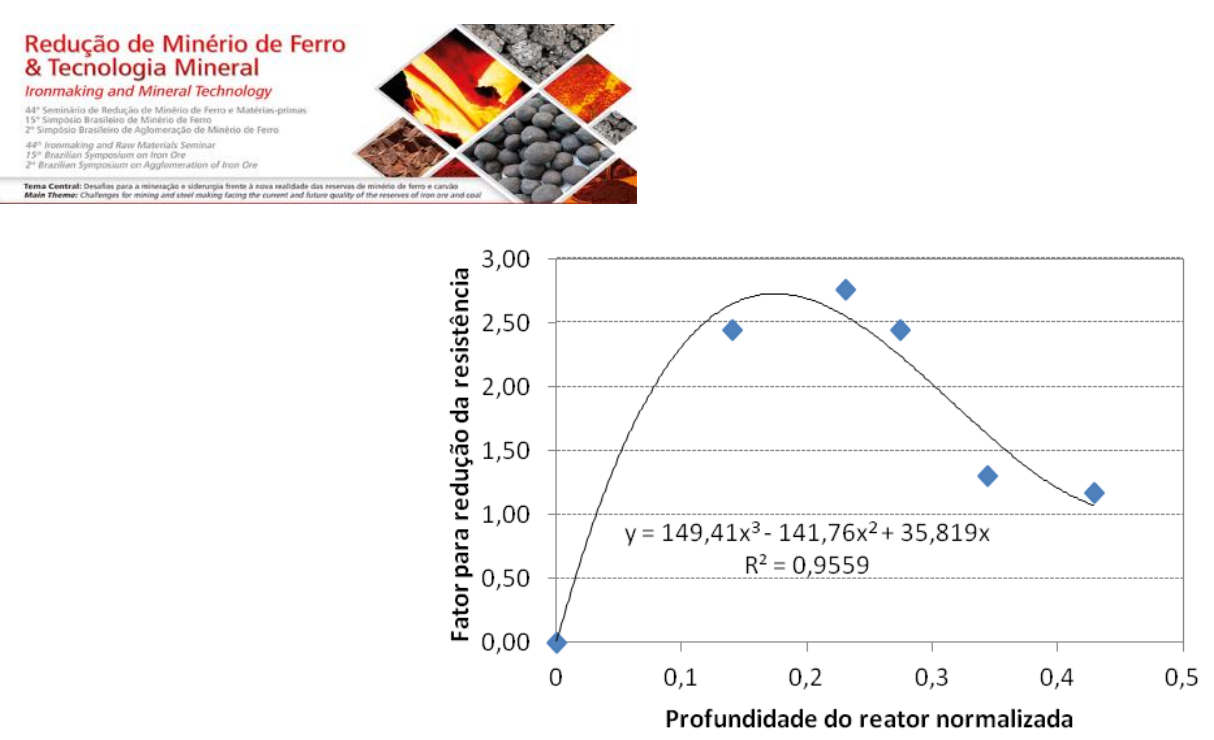

Figura 4 - Relação entre a profundidade normalizada estimada do forno e o fator de redução de resistência superficial.

Com bases na relação entre a profundidade normalizada estimada do forno e o fator de redução de dureza superficial calculado com pela redução da dureza superficial (Figura ), foi determinado o fator $\left(F_{a}\right)$ para ajuste da Equação 03 na região de redução. Desta forma tem-se a seguinte equação ajustada:

$$
\begin{array}{r}
F_{\alpha}=1+149,41 h^{\mathrm{a}}-141,76 h^{2}+35,819 h \\
k_{\alpha}=0,002 F_{\square} E_{k}^{1,162 a}
\end{array}
$$

na qual $\mathrm{h}$ é a profundidade do forno normalizada para $\mathrm{h} \leq 0,4$.

Como os dados experimentais obtidos no artigo referem-se a uma altura normalizada menor que 0,4 , para $h>0,4$ dentro da região de redução e das demais regiões do forno, foram consideradas válidas as seguintes equações:

$$
\begin{array}{r}
F_{\alpha}=1+1,17 \\
k_{\alpha}=(0,002 \times 2,17) E_{k}^{1,16}
\end{array}
$$

no qual o $F_{a}$ é recalculado com base no valor experimental de redução de dureza superficial para maior redução obtida por Huang et al. [7] equivalente a profundidade normalizada superior a 0,4 e o $\mathrm{k}_{\mathrm{a}}$ consiste na condição de saturação da Equação 07 para $h>0,4$.

\section{RESULTADOS E DISCUSSÃO}

Como apresentado, as pelotas de minério de ferro foram representadas como esferas de $12,5 \mathrm{~mm}$ de raio nas simulações em DEM de forma a diminuir o número de partículas e com isto diminuir o esforço computacional realizado durante o processo. Entretanto, na simulação da região 04 (região do quebrador inferior), utilizou-se esferas de tamanho médio $12,5 \mathrm{~mm}$ para representar as pelotas de tamanho comercial, a fim de avaliar o impacto desta variação de tamanho na análise dos resultados. Nesse caso, o número de partículas na simulação 150.000 ante 15.000 nas simulações com esferas de $12,5 \mathrm{~mm}$ de raio. Porém, não houve grandes alterações no perfil das energias dissipadas durante a simulação como pode ser observado pela distribuição acumulada (Figura 5). Por outro lado, as simulações do movimento das pelotas na região do quebrador inferior apresentaram um aumento em mais de $50 \%$ na média da energia especifica $(\mathrm{J} / \mathrm{kg})$ causado principalmente pelo

* Contribuição técnica ao 44 Seminário de Redução de Minério de Ferro e Matérias-primas, 15 Simpósio Brasileiro de Minério de Ferro e 2o Simpósio Brasileiro de Aglomeração de Minério de Ferro, 15 a 18 de setembro de 2014, Belo Horizonte, MG, Brasil. 
aumento da energia tangencial, possivelmente causada pelo maior movimento das pelotas de menor diâmetro. Mesmo com este aumento da média, conclui-se que as simulações usando pelotas de $25,0 \mathrm{~mm}$ conseguem representar grande parte da dissipação especifica de energia que ocorre no forno.

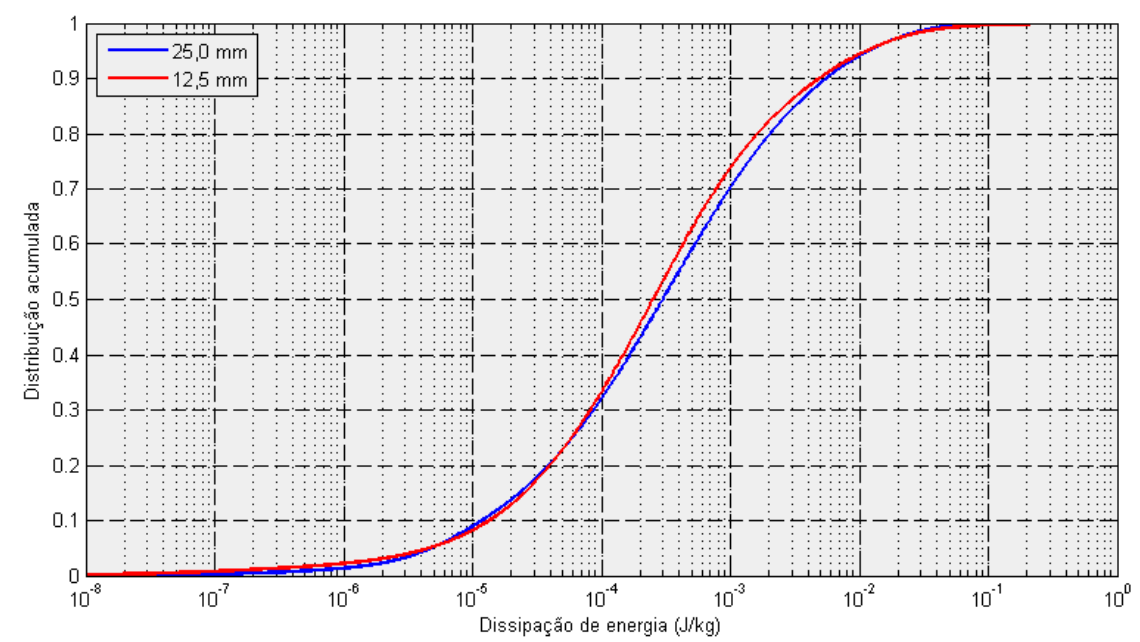

Figura 5 - Distribuição acumulada da dissipação da energia na região 04 como função do tamanho das pelotas nas simulações em DEM.

As dissipações de energia ocasionadas pelas colisões e sua respectiva geração de finos foram avaliadas em cada região sendo que a maior frequência de colisões é encontrada na região mais próxima ao fim da coluna de redução, mostrando que há um efeito da altura relativa das pelotas. Este efeito pode ser percebido na Figura , que ilustra a variação da média da dissipação de energia normal das colisões em função da altura e do raio do forno.

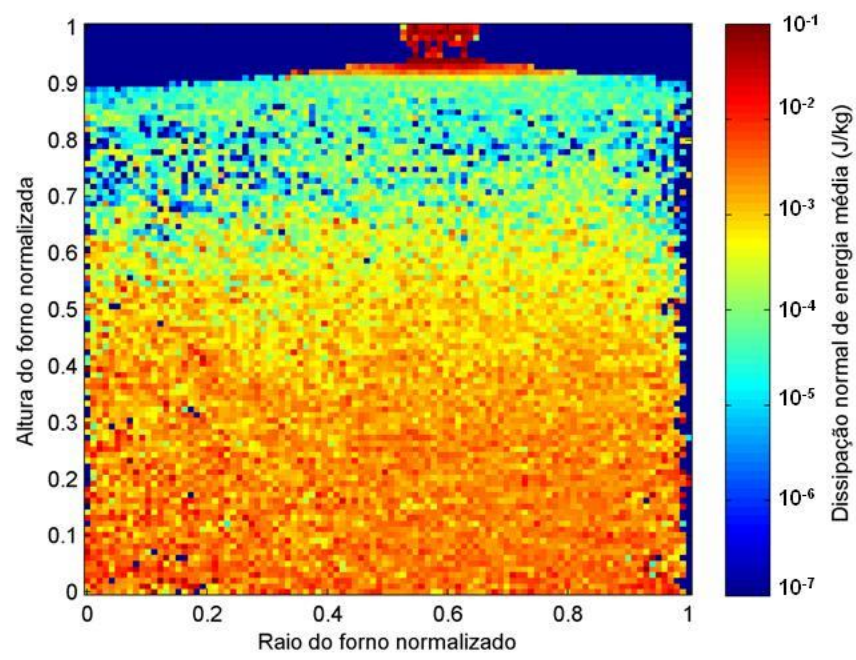

Figura 6 - Energia normal média transferida às partículas em função da altura e do raio do reator na região 01.

Pode-se verificar que as maiores dissipações de energia ocorrem na zona de redução. As regiões dos quebradores apresentam energias muito baixas, as quais não serão suficientes para fragmentar as pelotas por impacto, mesmo elas se encontrando reduzidas. Comparando a distribuição da energia de fratura de pelotas queimadas com 12,5 mm de diâmetro com as distribuições encontradas nas simulações, pode-se notar que não há energia para a fragmentação das pelotas

* Contribuição técnica ao 44 Seminário de Redução de Minério de Ferro e Matérias-primas, 15은 Simpósio Brasileiro de Minério de Ferro e $2^{\circ}$ Simpósio Brasileiro de Aglomeração de Minério de Ferro, 15 a 18 de setembro de 2014, Belo Horizonte, MG, Brasil. 
(Figura 7). Considerando que estas pelotas estão sendo reduzidas, sua estrutura está sendo fragilizada, resultando em perda resistência à compressão e ao impacto. No trabalho de Huang et al. [7] observou-se que com a redução ocorreu uma diminuição em aproximadamente $75 \%$ na resistência a compressão de pelotas. Presumindo que a energia de fratura seja reduzida da mesma forma, a energia dissipada na zona de redução ainda não seria suficiente para a fragmentação das partículas por impacto (Figura 7).

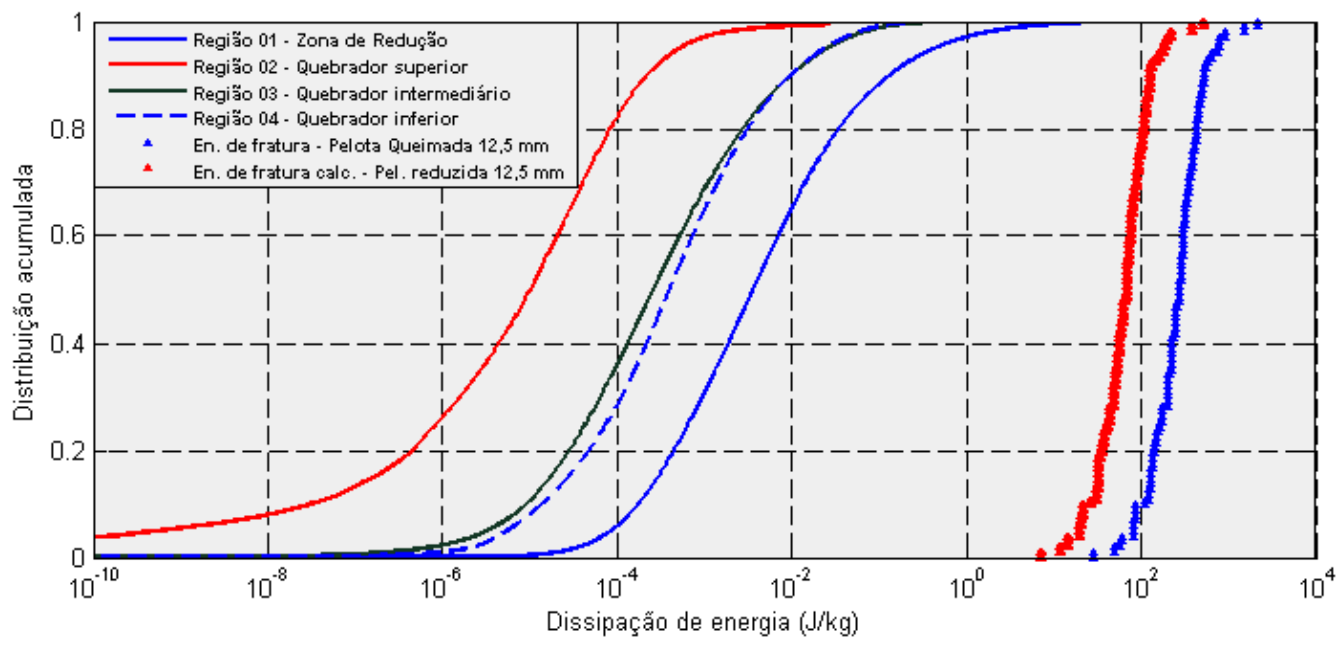

Figura 7 - Distribuição acumulada da dissipação da energia nas quatro regiões simuladas comparada a energia de fratura de pelotas queimadas de $12,5 \mathrm{~mm}$ e energia de fratura de pelotas reduzidas calculadas de $12,5 \mathrm{~mm}$.

A partir da Equação 04 foi possível aplicar as energias resultantes das colisões das pelotas de forma a estimar a proporção de finos gerados por abrasão, considerando que a suscetibilidade das pelotas à abrasão não seja alterada durante a redução. Para isso foi considerada apenas a geração de finos dentro do reator. Ao aplicar o modelo de abrasão encontra-se o valor médio de $1,19 \%$ de geração de finos ao final da região de redução. Observando-se a curva da taxa de geração de finos para velocidade padrão de descida da carga verifica-se que a geração de finos é mais acentuada próximo à base da região onde ocorre a maior dissipação de energia (Figura 8). A velocidade de descida da carga influencia na geração de finos. A simulação de um aumento de $50 \%$ e $100 \%$ na velocidade de descida resultou na geração de uma proporção de finos de 1,45 e 1,55\%, respectivamente. A geração de finos simulada a partir das colisões não se mostrou significativa nas regiões dos quebradores, tendo em vista as baixas magnitudes das energias de colisão nessas regiões. A porcentagem de finos produzidos nessas duas regiões somadas não ultrapassa $0,05 \%$ da massa analisada. 


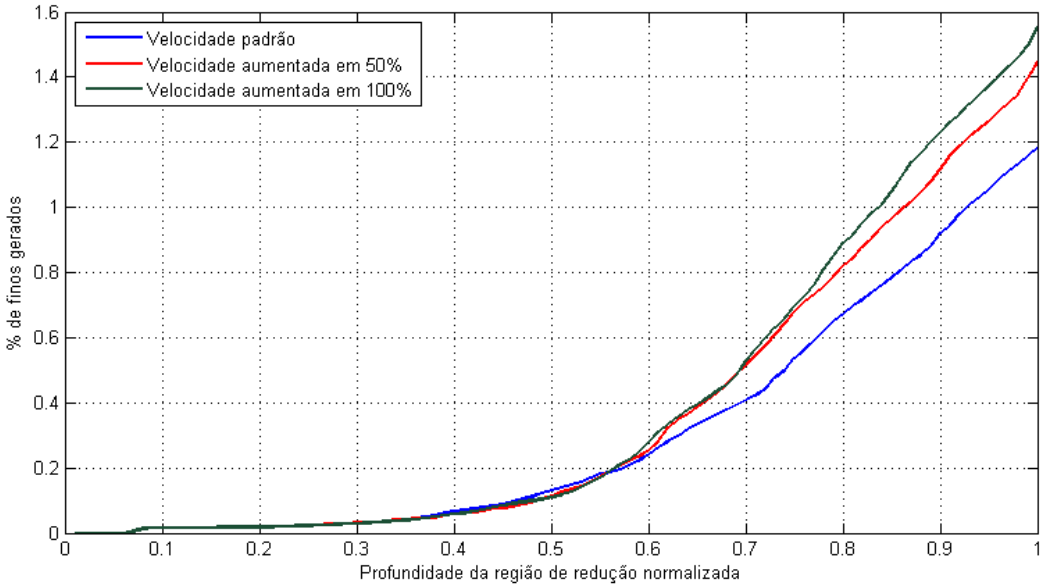

Figura 8 - Evolução da geração de finos em relação à profundidade do forno na região de redução.

Entretanto, esses valores não representam adequadamente o que ocorre no interior do forno de redução devido ao fato das pelotas sofrerem modificação em sua estrutura interna e perda da sua resistência física. A fim de levar em consideração esses efeitos, ou seja, aplicando a Equação 07 e 09, pode-se verificar que o valor da geração média de finos na região de redução passa a 2,55\% (Figura 9), o que representa um aumento na geração de finos por abrasão em quase $115 \%$. Assim, considerando este aumento, bem como a contribuição adicional da geração de finos nas regiões dos quebradores, o valor final previsto para a quantidade de finos gerados dentro do reator foi de $2,6 \%$. Se considerado esse valor para a geração de finos, a perda resultante seria de 16.950 t/ano aproximadamente. Este valor apresenta boa correspondência com aqueles observados em plantas industriais que produzem ferro esponja a frio e realizam peneiramento na descarga (2 a 4\%) [1]. É importante lembrar que o valor de referência mencionado apenas corresponde à descarga, de maneira que, a este, deve ser computados também os finos gerados dentro do reator como, por exemplo, o off take gas (fluxo gasoso emanado continuamente da zona de resfriamento), para correta comparação com o valor encontrado pelo modelo.

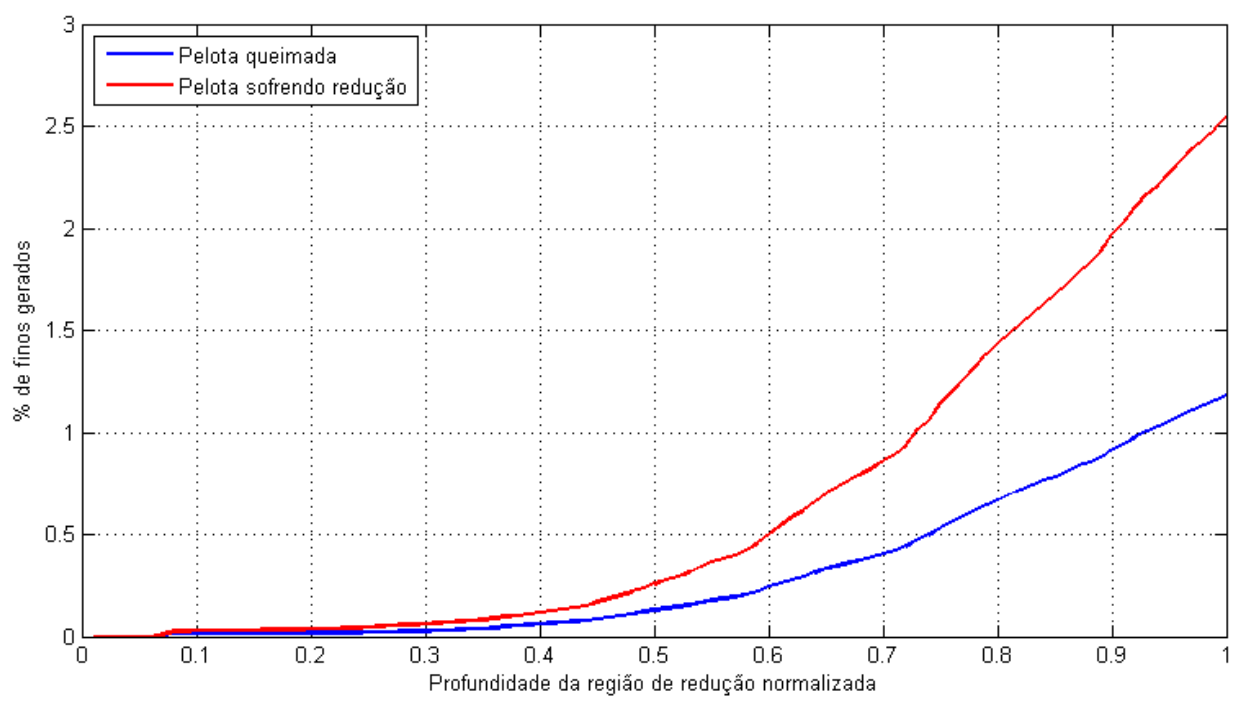

Figura 9 - Comparação da evolução da geração de finos em relação à profundidade do forno na região 02 com o efeito da perda de resistência causado pela redução.

* Contribuição técnica ao 44 Seminário de Redução de Minério de Ferro e Matérias-primas, 15은 Simpósio Brasileiro de Minério de Ferro e $2^{\circ}$ Simpósio Brasileiro de Aglomeração de Minério de Ferro, 15 a 18 de setembro de 2014, Belo Horizonte, MG, Brasil. 


\section{CONCLUSÃO}

A partir dos resultados das simulações com o software EDEM ${ }^{\circledR}$ do movimento de pelotas de minério de ferro em um forno MIDREX foi possível chegar às seguintes conclusões:

- A região de redução da carga, principalmente aquela mais próxima à injeção dos gases de redução, estará mais sujeita a dissipações de energia de magnitudes capazes de gerar finos de abrasão. As dissipações de energia das colisões têm influência direta da altura da coluna de pelotas. A mudança na velocidade de descida da carga resultou na maior dissipação das energias de colisões. Isto terá como consequência uma maior geração de finos por abrasão.

- Na região dos quebradores, a dissipação de energia das colisões das pelotas apresentaram baixas magnitudes. Devido a isso, pouca fragmentação por impacto é esperada nestas regiões. Por outro lado, deverá ocorrer a geração de finos por abrasão que não devem ser impactantes ao processo.

Assim, com o auxílio de simulações utilizando o software EDEM $^{\circledR}$ foi possível demonstrar a aplicação do método dos elementos discretos ao processo que ocorre no reator Midrex. Com base nos resultados encontrados, aplicando-se modelos conhecidos, como por exemplo, o modelo de abrasão de minério de ferro desenvolvido por Tavares e Carvalho [4], foi possível estimar a geração de finos no interior do reator como sendo da ordem de 1,2 a 2,6\%, dependendo das premissas utilizadas. Esses valores estão compatíveis com aqueles encontrados em plantas industriais.

\section{Agradecimentos}

Os autores gostariam de agradecer a Vale SA por patrocinar a pesquisa e por dar permissão para publicá-lo. Os autores também agradecem DEM Solution pelo uso do software EDEM como Programa Acadêmico. LM Tavares também gostaria de agradecer ao Conselho Nacional de Pesquisa (CNPq) e RM de Carvalho também gostaria de agradecer a CAPES pelo apoio ao trabalho.

\section{REFERÊNCIAS}

1 Midrex. Direct from Midrex - 3rd / 4th Quarter 2011. Midrex Technologies, Inc., p. 9, 2011. Disponível em < http://www.midrex.com/uploads/documents/DFM\%203rd4thQtr\%202011.pdf> Acesso em 15/08/2013

2 Midrex. Shaft Furnace Technologies - Solutions for Steelmakers. Midrex Technologies, Inc., p. 15, 2012. Disponível em < http://www.midrex.com/uploads/documents/MIDREXShaftBrochure2.pdf> Acesso em $10 / 01 / 2013$

3 Midrex. World direct reduction statistics. Midrex Technologies, Inc., p. 14, 2013. Disponível em <http://www.midrex.com/uploads/documents/MDX\%20STATS\%202012\%207-313Final.pdf> Acesso em 02/01/2014

4 Tavares LM, Carvalho RM. Modeling ore degradation during handling using continuum damage mechanics. International Journal of Mineral Processing, 2010; 101(1-4): 21-27.

5 Carvalho RM, Tavares LM, Barrios GKP, Kwade A. Contact parameter estimation for DEM simulation of iron ore pellet handling. Powder Technology, 2013; 248: 84-93. 
6 Carvalho RM. Mechanistic Modeling of Semi-Autogenous Grinding, Ph.D thesis, Universidade Federal do Rio de Janeiro, 2013

7 Huang Z, Yi L, Jiang T. Mechanisms of strength decrease in the initial reduction of iron ore oxide pellets. Powder Technology, 2012; 221: 284-291.

8 Parisi DR, Laborde MA. Modeling of counter current moving bed gassolid reactor used in direct reduction of iron ore. Chemical Engineering Journal, 2004; 104(1-3): 35-43.

* Contribuição técnica ao $44^{\circ}$ Seminário de Redução de Minério de Ferro e Matérias-primas, 15o Simpósio Brasileiro de Minério de Ferro e 2o Simpósio Brasileiro de Aglomeração de Minério de Ferro, 15 a 18 de setembro de 2014, Belo Horizonte, MG, Brasil. 